\title{
FOURTH DISCUSSION SESSION
}

(Friday morning; 8 September, 1972)

(following the review paper by $\mathrm{K}$. O. Wright)

\section{Chairman: O. C. WILSON}

O. C. Wilson: We thank Dr. Wright for his well-presented review, and invite ques. tions and discussion of his paper.

Bolton: I have some comments and a question. First, in regard to these reverse P Cyg profiles in the spectrum of $\theta$ Ori $\mathrm{C}$, I think the situation there is not quite so simple as Conti seemed to believe in his publication. Walborn at David Dunlap Observatory has some lower-dispersion spectra that show a 'regular' P Cyg profile. Therefore, we cannot be dealing with simple infall of matter and the relationship to YY Ori stars is tenuous at best. Second, it has been my impression from reading the literature recently, particularly the works by Mihalas and collaborators, that the Bowen fluoresence mechanism does not give an adequate explanation of the Of-star emission but that these phenomena find a natural explanation in the non-LTE reference frame. In connection with this, Mihalas* has shown that some of the emission features are luminosity sensitive. Both Walborn (1971) and Conti and Alschuler (1971) have recently given luminosity classifications for the O-type stars, and they both define a class of supergiants. In view of Dr. Wright's comments on the O-type supergiants in his review, I would like to hear his and Dr. Underhill's opinion of this classification.

Underhill: Regarding reverse P Cyg profiles, they have emission to the violet of the absorption. They are not very common, but neither are they very rare. For instance, I've obtained spectrograms showing $\mathrm{H} \alpha$ for a good many B-type supergiants from time to time. One time the emission is a bit stronger on the red side, another time on the violet side of whatever absorption there is. Generally, the lines are pretty well filled in and you really don't see anything - just a little bump if you make intensity tracings. The simple, uniform model is either an expanding or a contracting atmosphere, but, in fact, the atmosphere changes and the whole $\mathrm{H} \alpha$ line is neither absorption nor emission, but just slight undulation up or down on one side of the nominal profile. We have a more complex situation than a simple uniform expanding sphere, although what Conti says is right as a very first order of approximation.

About the supergiant early O-type stars, how do you define a supergiant? It's quite easy for late-type stars for which there is a large difference of magnitude between stars that are very bright and have a characteristic type of spectrum, called supergiants, and those that are on the main sequence. There are the stars called giants too: they are all separate. But once you consider the O-type stars, those on the main sequence

\footnotetext{
* See Hummer's discussion on p. 277.
} 
have $M_{v}$ about -4 , maybe as bright as -5 , while supergiants, no matter what type they are, are usually of $M_{v}$ only about -6 or -7 . The uncertainty in determining the absolute magnitude is so great that it is very hard to say, at type 05 , that you have isolated different absolute magnitude groups. I think the terms 'supergiant' and 'main-sequence' are hardly relevant considering the uncertainties in the distances of these early O-type stars. Some of these stars show evidence that their atmospheres are expanding a little more than those of others, but I would hesitate to use the term 'supergiant' because it has so many other connotations for the later-type stars. In any case, the atmospheres are so expanded that you have to consider non-L.T.E. physics - real physics. You cannot argue from the equations of thermodynamic equilibrium.

O. C. Wilson: I gather that, in a sense, what you're saying is that the spectroscopic criteria are just not sharp enough?

Bolton: I don't want to let that comment go by without challenge. The spectroscopic criteria are certainly quite sharp. I have seen the spectra, and even I would have no trouble classifying the stars. I think that there can be no doubt that the spectroscopic classes of Walborn and Conti are real. Furthermore, interpretation of a luminosity effect does not require that one invoke LTE, or non-LTE, or magic. One must simply get distances or sometimes only relative distances. I don't want to minimize the difficulties involved in this, particularly the difficulties in deriving absolute magnitudes, but one frequently finds O-type stars of two or more classes in the same cluster. Then the photometry gives the luminosity differences directly and easily. It is true that spectroscopic binaries can complicate the interpretation, but they cannot account for more than 0.75 , and a range of at least three magnitudes is observed.

Underhill: You can do this. I don't quibble with that, but to attach a physical meaning to the class is the difficult step.

Thackeray: As to the absolute magnitudes of the O-type stars, may I suggest that a little more work on the Magellanic Clouds will give us a pretty safe answer whether the luminosity of an $\mathrm{O} 5$ star can reach anything like that of a later-type supergiant. Our work in Pretoria, scratching the surface, didn't show up any O-type star brighter than $m_{v}=12$ or 12.5 , whereas A-type stars are known with $m_{v}=9.5$ or 9.2 . We are doing more work in Pretoria, but I think it's very unlikely that we shall turn up an O5 star brighter than about $12^{\mathrm{m}} .2$.

Popper: I'd like to ask a question about the so-called reverse P Cyg profiles, such as are found in the spectrum of $\theta$ Ori $\mathrm{C}$. What are the actual velocities? Is the emissionline or the absorption-line velocity that of the star? To interpret the profiles, you must have some picture of what the velocities are, and, if there is a change, what it is that is changing.

Wright: Conti (1972) found that the absorption was redward displaced, with some variation, and the emission was violet-displaced.

Popper: It seems to me that we are not seeing infall of matter, and then outfall, but that we are seeing an expanded shell with a changing distribution of matter in it. Sometimes the part behind the star is stronger. 
Underhill: This is the impression one gets from $\mathrm{H} \alpha$ in the spectra of B-type supergiants.

Wright: I was really trying to emphasize, in my review, that the reverse $\mathrm{P}$ Cyg profiles have not been discussed much in the literature until very recently. The hydrogen lines in the spectrum of VV Cep can be at least partly explained as the sum of red and violet components of emission; that is, when I was determining the secondary spectrum, I had to take into account both the violet and the red sides to determine the mean profile. There is also a component at about $-60 \mathrm{~km} \mathrm{~s}^{-1}$, which is always present and which I think is in the system itself. But I don't think we need worry very much about infall for most of these stars. I think it's a natural phenomenon that may well be at least partly explained, as Dr. Underhill suggested, by random motions in their atmospheres.

Bolton: I am not convinced that early-type supergiants appear only in binary systems of the VV Cep type. I have seen classification spectra of B-type supergiants in which the lines are clearly doubled.

Underhill: That is an extremely rare observation.

Bolton: I'm not sure I agree. I know of several examples, and I don't see any theoretical reason why binary systems containing two early-type supergiants should not exist. The surface gravities indicate that an early B-type or O-type supergiant has not expanded by a factor of more than three or four. If they start out in reasonably detached systems with periods of a few days (say five or more) they would not begin to interact until one of the stars became of spectral type B5 or thereabouts. Furthermore, the range in absolute magnitudes from the main-sequence to supergiants is small enough, for early-type stars, so that the probability of observing a two-spectra supergiant system is much higher than it is for later types.

Huang: Dr. Underhill raised a very interesting and fundamental problem just a moment ago - about the high turbulent velocities derived from curves of growth. In a recent paper in Nature (Worrall and Wilson, 1972) it was argued that these turbulent velocities do not exist. Anyway, they are very strange, because they show that within an optical thickness of unity the velocity reaches a high supersonic value. Perhaps these high values for the turbulent velocity are the result of a lot of shocks in the envelope. I used the illustration in my review paper of people smoking in a room; jet streams come up to collide with the envelope, and I identify this envelope with the extended atmosphere. Now, when each jet stream interacts with the medium, it produces a shock. So there are a lot of shocks around to give the large curve-of-growth velocity. Do you think this is possible?

Underhill: It's a very nice idea to try to account for the broadened line profiles by the velocity fields created by shocks. But the problem of microturbulence is fundamental to the theory of curve of growth. I think the real point is that it uses the wrong theory for line formation. One obtains a curve of growth by assuming a certain relationship, between the absorption coefficient and the equivalent width of a line, that is based on some theory - elementary or otherwise. If you use pure absorption so-called theory, you obtain one curve of growth, while scattering theory gives you 
another. For the same number of atoms, scattering theory gives a higher flat portion of the curve of growth than does absorption theory - yet you started off with the same model and just used a different approximation to radiative transfer. Because the central part of the curve of growth is higher than expected from absorption theory, you deduce microturbulence. Actually, you used the wrong transfer theory to describe the formation of absorption lines. For these extended atmospheres, very simple physical arguments show that a scattering theory is much more relevant to the understanding of transfer than is an absorption one, and I think that practically all microturbulence results are figured out with reference to the absorption-type curve of growth. All this has been pointed out by Menzel (1939).

O.C. Wilson: We are talking here, I think, about things like $\zeta$ Aur and $32 \mathrm{Cyg}$ which have always seemed to me the interstellar case of a star, an apparently small object, shining through some material. In that case it seems to me that the simple absorption theory of transfer is perfectly adequate.

Underhill: You can't argue that way. You're assuming that the populations of the absorption levels have a Boltzmann distribution. Actually, if you consider the radiative field, you may find that in these stars the atoms are preferentially packed down in the lower levels. If you assume a Boltzmann distribution to derive an effective number of atoms from the equivalent width, you just get problems, and I think the net result in a straightforward curve-of-growth measurement is the deduction of a large microturbulent velocity. This is just a fictitious quantity, and there are so many ways that one can account for it being positive. If one found a negative microturbulence, one would really worry - yet there's no obvious reason why one shouldn't find negative shifts. The approach to a physical understanding is complex, and I would be the first to say that what I am saying is far too simple.

O. C. Wilson: Couldn't it be that these displaced components of the $\mathrm{K}$-line that Ken Wright showed us arise from the large elements in a hierarchy of all possible sizes of cells? The little fellows, all added up together, give us what we think is microturbulence, because they affect the line-absorption coefficients. Big elements often have nothing to do with the line-absorption coefficient: you see them individually. If you have a hierarchy of all possible sizes, presumably many more small ones than big ones, then it seems to me that the problem/reduces essentially to the simplest case.

Underhill: 1 don't think we can answer this definitely one way or the other. My studies of physics incline me to reject entirely the hypothesis of microturbulence and to consider the non-LTE situation.

O.C. Wilson: That is a noble endeavour, but it's likely to be a difficult one. Do we have any further comments?

Batten: Going back to Dr. Huang's comment, I would like to ask whether shock waves between freshly ejected matter and the surrounding medium could produce discontinuous velocities in the circumstellar matter that would be observable as the steps in velocity curves, to which I referred in my review.

Huang: There will be no discontinuity of any kind if you have a lot of shocks 
around. They are smoothed out. That is why I propose a large number of shocks in the medium.

R. E. Wilson: I was curious about the values given for the individual masses of $\varepsilon$ Aur, since this is single-spectrum binary.

Wright: They were based on some calculations that Stephen Morris made a few years ago. He estimated the absolute luminosity of the primary component in several ways, and then assigned a mass to it on the basis of evolutionary tracks (which run almost at constant luminosity for so massive a star). From the mass function it was then possible to compute pairs of values for the mass ratio and orbital inclination, and to derive from these values of the radii of the two components and hence (from the spectral type) of the absolute luminosity of the primary star. The values of the masses quoted are those that give an estimate for the last-named quantity consistent with the initially assumed value. Neither Morris nor I claim that the derived masses are reliable to all the significant figures quoted.

Fracastoro: It appears that the atmospheres of $31 \mathrm{Cyg}$ and $\zeta$ Aur are not uniformly distributed even during the same eclipse - I'm thinking of the larger-scale clouds. I would also like to emphasize that it appears in general that the advancing hemisphere of the expanded atmosphere is more developed than the receding one. That's one thing that probably Otto Struve was the first to point out.

Wright: I'm not quite sure whether that is true or not. By 'more developed', do you mean 'more extended'? Then you would expect that there would be more elements in the advancing side and therefore we would observe more random velocities. Is that what you mean?

Fracastoro: No. I think that the ingress phase lasts longer than the egress.

Wright: Oh, I see. I think the data that I had on $\zeta$ Aur and that I published in my Vistas article (Wright, 1970) show the ingress and egress phases to be fairly symmetrical. I'm thinking of $31 \mathrm{Cyg}$ and $32 \mathrm{Cyg}$, as well as $\zeta$ Aur, the last-named certainly varies. There is no question the 1937 eclipse indicated a greater extension than did any of the others. For $32 \mathrm{Cyg}$, however, if anything, the extension was a little bit larger at egress than at ingress. You would have to look at the data; there is scatter from one eclipse to the next, and the result is not too clear-cut.

Fracastoro: You showed a diagram of the intensity of the chromospheric K-line, in the spectrum of $\zeta$ Aur, as a function of time. All the curves seem to be higher on the right-hand (egress) side than on the left.

Wright: For $\zeta$ Aur, the Cambridge observation of the ingress of the 1937 eclipse showed greater intensities than have been observed at any other eclipse. In general, the intensities of the chromospheric lines in the spectrum of $\zeta$ Aur seem to be a little greater during ingress than during egress, for equal times from mid-eclipse. The reverse seems to be true for $32 \mathrm{Cyg}$, but the difference is not great.

Herczeg: I always thought this was explicable mainly in terms of the orbital eccentricity, the velocity of the tangential motion being higher before than after the eclipse. I would also like to ask Dr. Wright about the chromospheric lines seen in the spectrum of $\varepsilon$ Aur near its eclipse. You mentioned these in your review, can you tell 
us more? Because of our utter ignorance of the nature of the secondary component, every bit of information may turn out to be quite important. My question is perhaps too naive. Can we find out in this way something about the nature of the object? Is it a star, or are the chromospheric absorption lines just relatively narrow additional absorption lines originating in a gas cloud?

Wright: The real problem about the chromospheric absorption lines in the spectrum of $\varepsilon$ Aur, if I can answer this first, is that we don't really know what we are observing. They were easily observed in the 1955-1956 eclipse. I obtained numerous observations of them, and so did others (Struve and Pillans, 1957; Hack, 1957; Wright and Kushwaha, 1957). The chromospheric lines really looked quite similar to those in the spectrum of $31 \mathrm{Cyg}$, but, according to the radial-velocity curve the observed eclipse is the 'secondary' eclipse for $\varepsilon$ Aur, whereas it is the primary eclipse for 31 Cyg and $\zeta$ Aur. We know so little about the system that theories of black holes, etc., are perhaps a little premature. The sharp lines are certainly present in the spectrum, but it seems probable that they are produced in some disk or other feature, and are not true chromospheric phenomena. Just what mechanism produces them, what the source behind the absorbing material is, we don't know yet - at least, not in my opinion. Concerning this eccentricity effect, it is appreciable, but I think it is not great enough to account for the effects observed in the spectrum of $\zeta$ Aur in 1937. However, the time of periastron is such that the intensities at a given phase could be greater during ingress for $\zeta$ Aur, and during egress for $32 \mathrm{Cyg}-$ as I have just suggested.

Bolton: Have there been any investigations of the rapid variability of the spectra during these atmospheric eclipses? If there really are chromospheric phenomena such as you find in the Sun, where the life-times of spicules, and so on, are typically about a few minutes, then one might expect to find variations in the line profiles, and in the velocities, on a time scale of hours, in these more extended atmospheres.

Wright: Dr. Odgers at Victoria has for many years attempted to obtain a number of plates of these objects - particularly of $31 \mathrm{Cyg}$ - during a night. There have been slight indications of changes during a night, but they have been quite small. Over a twenty-four-hour period, however, there is no doubt that real changes occur. We are not sure what happens within a few hours, and rapid-scanning techniques will be important in helping to find out. We tried this with the Isocon scanner of the University of British Columbia last October and there were just marginal effects when we were getting a scan every fifteen seconds or so, but even when we integrated several series of 44 traces over a couple of hours, the effects were still marginal. The resolution was not quite good enough.

R. E. Wilson: Are these chromospheric features that you describe in the spectrum of $\varepsilon$ Aur the same ones that were described by Kuiper, Struve, and Strömgren (1937)? They described satellite lines that appeared around primary eclipse.

Wright: Yes, they are the same.

R. E. Wilson: What did you mean when you said the lines were seen around secondary eclipse? There is only one eclipse, so it has to be the primary. 
Wright: For $\varepsilon$ Aur, $u=90^{\circ}$ at the time of eclipse. For the other stars, $u=270^{\circ}$.

R. E. Wilson: Alright. These lines show a velocity reversal between the ingress and the egress of the eclipse, so there has to be either a rotating object or an orbiting object present. An orbiting object seems more likely, so it seems unusual to call the lines 'chromospheric'. They are absorption lines, of course, not emission lines?

Wright: They are absorption lines, and they are quite sharp. They are produced in the outer chromosphere because radiation from the B-type star passes through the chromosphere. As the eclipse progresses, the lines become stronger, and are produced in the inner chromosphere, or finally the photosphere - if you want to call it that. This happens before second, and after third contacts, so there should be some rotation effect but it is not large, and the random motions are greater.

R. E. Wilson: But for $\varepsilon$ Aur, as described in 1937, there was a progressive velocity shift from the time the lines were first seen, until they disappeared. This is exactly what you would expect if you were seeing Keplerian motion of orbiting material. At egress the shift had the opposite sense from that seen at ingress. The lines were in absorption. So it does look as if the absorption was caused by orbiting material rather than by chromospheric phenomena.

Wright: The results for the 1955 eclipse of $\varepsilon$ Aur have not been published in complete detail. The velocities of the 'chromospheric' lines were positive during ingress and negative during egress, as shown in Figure 11 of my Vistas article, (Wright, 1970). I am not sure whether it really is a rotation effect, and it may not be a chromospheric effect, since we do not know the nature of the object that causes the eclipses of $\varepsilon$ Aur.

$R$. E. Wilson: It not only looks like a rotation effect, but like a progressively varying rotation effect, as if the line of sight were passing through material for which the Keplerian velocity is a function of distance from the centre of the object.

O. C. Wilson: If there are no further comments on the review paper, we will ask Lloyd Evans to speak to us on "Binaries with Giant Primaries Showing Strong Ca II Emission."

Lloyd Evans: This group of about ten known members shows the following general properties:

(1) Single-spectrum binary, spectral type G-K, II-IV.

(2) Orbital period usually $\sim 20 \mathrm{~d}(17-\sim 80 \mathrm{~d})$.

(3) The Call emission is very strong and in some cases is found to consist of the normal, broad, double-peaked component with a superposed sharp component. The velocity derived from the sharp component follows the orbital radial velocity fairly closely, indicating that the emission region is close to but possibly not coincident with the primary.

(4) The primary is probably close to filling its Roche lobe.

(5) HD 209813 and $\lambda$ And show semiregular light variability with a period not equal to the orbital period and much greater than the period for radial pulsation.

A new example is HD 158393, G5 III : $m_{\mathrm{pg}}=9.3$. This was found to have strong Call emission and proved to be a single-lined spectroscopic binary with $P=30$.9. This star shows several interesting points: 
(1) The absorption lines are very broad. If interpreted as rotational broadening, $v \sin i \sim 150 \mathrm{~km} \mathrm{~s}^{-1}$.

(2) CaII emission is also broad. This may indicate it arises from a region sharing the supposed rotation of the photosphere.

(3) Call emission radial velocity follows the orbital velocity but with a slightly larger amplitude. This could arise, on the present model, from a non-uniform distribution over the rotating disk.

(4) There is a suspected $10-\mathrm{d}$ periodicity in the residuals of radial velocities from the orbital solution, which exceed the measuring errors.

This work was done in collaboration with B. Emerson of H. M: Nautical Almanac Office.

Plavec: I think this is a very interesting group of stars, although I don't know exactly quite which stars you include in this group. From what you mentioned, I think you mean also, for example, $\lambda$ And or $\zeta$ And, but these are single-line spectroscopic binaries. Therefore I would like to ask you what is the basis for your statement that the primary component fills the Roche lobe?

Lloyd Evans: This is based on rather rough ideas, discussed in Herbst's unpublished thesis. One simply obtains the orbit and makes some guess as to the size of the star and what mass one would need for the companion.

Plavec: But we don't observe any traces of the secondary spectrum?

Lloyd Evans: Certainly not in the one I've studied.

Plavec: Otherwise the system or the group might be related to relatively shorterperiod eclipsing binaries of the type of AR Lac and other stars with pronounced calcium emission in their spectra. There is no doubt that in the spectra of $\lambda$ And and $\zeta$ And, the emission lines of calcium are extremely strong.

O. C. Wilson: Sanford (1951) worked on AR Lac many years ago. There are two emission components at $\mathrm{K}$ and their velocity shifts indicate that they arise from something moving with the two stars. They look more or less normal, I thought. I also have a number of plates of $\lambda$ And at $10 \AA \mathrm{mm}^{-1}$. At even higher dispersions there is a very tiny central absorption, the width of which changes during the orbital period.

Biermann: This star HD 158393 seems very interesting because its rotational velocity seems very high for a late-type star. If you accept the figure at its face value, it would indicate that when the star was on the main sequence, its rotational velocity exceeded the break-up velocity, and therefore the angular momentum must have come from somewhere else. Can the profiles be explained by anything other than rotation?

Lloyd Evans: The star should certainly be studied at high dispersion, in particular to determine the line profiles. I am not prepared to say, from my plates at $49 \AA \mathrm{mm}^{-1}$, that the profile shape is what you would expect from rotational broadening.

Cowley: As most of you probably know, there is a large project going on at Michigan to classify all of the southern HD stars, and the plates have all been taken with the Michigan Schmidt. In the course of this, we found several stars of this type, which perhaps should be looked at in more detail; perhaps some of them will turn 
out to be eclipsing or we may even find a second spectrum. If anyone is interested, I could send them a list of the names. Nothing is known other than that they are $G$ or $\mathrm{K}$ giants that have strong $\mathrm{H}$ and $\mathrm{K}$ emission; too strong for normal stars.

Bolton: We have a number of spectrograms of various stars of this type at Toronto. They were obtained at $12 \AA \mathrm{mm}^{-1}$ by two graduate students - Herbst and Campbell. I've looked at these, and every one of the spectra I've seen shows very fuzzy broad lines which, interpreted as rotationally broadened lines, give velocities of the order of $100 \mathrm{~km} \mathrm{~s}^{-1}$. They're absolutely the worst line profiles I've ever seen in a K-type spectrum.

Wright: How were these stars chosen?

Bolton: I'm not sure. I believe that they were picked from various lists of stars for which there is evidence of photometric and radial-velocity variations with different periods.

Andersen: Are you going to study HD 158393 at high dispersion, or is it free for anyone?

Lloyd Evans: It is unlikely that I shall study it.

Hall: As Dr. Plavec said, there is some similarity between these binaries and those like AR Lac and RS CVn. Both groups of binaries show very strong $\mathrm{H}$ - and $\mathrm{K}$-emission lines in their spectra, and at least one component is of spectral type $\mathrm{G}$ or $\mathrm{K}$. I have already proposed that the RS CVn binaries are in a pre-main-sequence phase of evolution. These other systems can also be understood in the same way. They contain relatively luminous stars (between luminosity classes III and II), whereas the components of a typical RS CVn system are less luminous (around class IV for the larger and between IV and V for the smaller). The components in RS CVn systems do not seem to be rotating rapidly, rather they seem to be rotating approximately in synchronism with the orbital motion. Perhaps these more luminous systems are still higher on the Hayashi track, and are rotating rapidly because of their youth (as some people say the T Tau stars are doing). As they get older, the rotation of the stars in these systems will also become approximately synchronized with orbital motion. How many of these stars are rotating rapidly? Are there some which are not doing so?

Lloyd Evans: It's news to me that the others in the group are rotating rapidly. I have material only for HD 158393.

Bolton: Those of which I have seen spectra have very poor line profiles, as though the stars are rotating rapidly. The best line profiles I've seen are in the spectrum of HD 209813, and even they are none too good.

Thackeray. Can Anne Cowley obtain rotational velocities from the Michigan material? Is anyone attempting a statistical study, such as Struve himself undertook in the 1930's to see if the distribution of orientations is random, as it should be if the profiles are rotationally broadened?

Cowley: Our dispersion is $110 \AA \mathrm{mm}^{-1}$. We can say if the line profiles are fuzzy, but we can't really say anything about the rotational velocities. Somebody could take our list and observe with higher dispersion.

Lloyd Evans: I had wondered, actually, whether HD 158393 might be a pre-main- 
sequence star. It does lie in the region of the Sco-Cen association. When we have a better idea of the radial velocity from the computed orbit, it may be possible to say whether or not it is an association member.

$O$. C. Wilson: If these stars are going to be interpreted as pre-main-sequence objects, it seems rather strange to me if they all turn out to be single-line spectroscopic binaries. We need more statistics.

Cowley: And their distribution should certainly tell us something, too.

Lloyd Evans: Another point is that young stars of late type usually have strong lithium lines in their spectra. At least one of the other stars in this group has been observed by Bob Fosbury of the Royal Greenwich Observatory, and he finds no strong lithium line in its spectrum.

Oliver: Perhaps all the known stars in the group are binaries because the strong $\mathrm{H}$ and $\mathrm{K}$ emission is a consequence of the binary nature.

Catalano: Fernie, Hube, and Schmidt (1968) have pointed out that HD 209813 is a high-velocity star. I think it cannot be a pre-main-sequence star. It would be interesting to determine the masses of its components: it is one of few high-velocity stars we know of to be double.

Popper: I have looked without success for the lines of the secondary component.

Plavec: Maybe we are talking about a group of stars that actually have very little in common. The lines in the spectra of $\lambda$ And and $\zeta$ And are certainly not broad nor indicative of high rotation: they are very nice and sharp. As for AR Lac, and other binaries of this group, a difficulty in their interpretation is that they do not fill the critical Roche lobe. So maybe the stars we are talking about belong to several groups, with only the strong emission at $\mathrm{H}$ and $\mathrm{K}$ in common.

O. C. Wilson: Which, however, may be stimulated by the presence of a companion.

Underhill: It would be very interesting to observe the $\mathrm{Mg}$ II resonance lines in the spectra of these stars. Those lines should be extremely strong, and if we ever get a satellite up capable of observing that region of the spectrum, I think it would be interesting.

O. C. Wilson: Well, we're counting on you, Anne!

Hall: Dr. Ca alano remarked that HD 209813 is a high-velocity star. A student of mine, Robert Montel, has examined the galactic distribution of all the RS CVn binaries we cou'd find. We looked at the distributions of the distances from the galactic plane, and of the veloc ties perpendicular to that plane. The average distance from the plane is about 80 parsecs, and the average velocity (perpendicular to the plane) is approximately $10 \mathrm{~km} \mathrm{~s}^{-1}$. Both these figures suggest an age somewhat less than $10^{8}$ yrs. One slight problem is that evolutionary tracks suggest the stars are even younger. Most of the individual components of RS CVn binaries fit on to pre-main sequence evolutionary tracks, calculated for single stars by Iben (1965), at points corresponding to ages of about $10^{7}$ yrs. Perhaps we should not try to fit components of binary systems onto tracks computed for single stars. Nevertheless, the RS CVn binaries seem to be young - I think younger than $10^{8} \mathrm{yrs}$. We haven't looked at the 
galactic distribution of the group of stars discussed by Lloyd Evans. It could be different.

$O$. C. Wilson: Then you make the RS CVn stars a little younger than the Hyades.

Biermann: Iben's pre-main-sequence tracks are a bit out of date. Larson (1969) showed that there is no Hayashi phase of any importance in the pre-main-sequence evolution of higher-mass stars.

Hall: These stars are around one solar mass.

Biermann: Then you can probably use Iben's tracks, but you always have to be careful when using single-star evolutionary calculations for components of binaries. You probably know that Larson's newer calculations show that you get a shell around stars, and I have heard from R. H. Koch that he looked at the possibility of finding binaries in the pre-main-sequence phase. He couldn't find any, because, as Larson showed, the shells obscure the central stars and hide the binary characteristics.

$O$. C. Wilson: Now we have two people who want to speak about $\beta$ Lyr. Dr. Faraggiana will give a preliminary report on observations by Hack and Cester, and Dr. Herczeg will also give a brief report.

Faraggiana: Dr. Hack obtained high-dispersion spectrograms of $\beta$ Lyr (12.4 and $7 \AA \mathrm{mm}^{-1}$ ) in both the blue and red regions of the spectrum during the 1971 international campaign. Radial velocities determined from the lines of Si II agree well with those obtained by earlier observers. Measures of the shell lines do not agree so well. The radial velocity obtained from the $\mathrm{H} \alpha$ absorption core is systematically more negative by $20 \mathrm{~km} \mathrm{~s}^{-1}$ to $40 \mathrm{~km} \mathrm{~s}^{-1}$ than the velocity obtained from the $\mathrm{H} \beta$ core. The emission peaks appear to be part of a single emission cut by the absorption core. The half width of the emission at $\mathrm{H} \alpha$ is about $450 \mathrm{~km} \mathrm{~s}^{-1}$. The radial velocity of the emission may be constant, or slightly variable. The profiles of lines in the red region also differ from those reported by Sahade (1964). The cores of the non-metastable lines of $\mathrm{He} \mathrm{I} \lambda \lambda 5876$ and 6678 vary in quadrature with the orbital velocity curve; while the metastable line of $\mathrm{He}$ I $\lambda 5016$ shows an almost constant velocity of $-120 \mathrm{~km} \mathrm{~s}^{-1}$. In general, lines originating in the stellar photosphere give velocities in agreement with those found by earlier observers, while lines originating in the envelope or streams do not.

During the same period, Dr. Cester obtained $U, B, V$ light curves, and light curves with the narrow-band interference filters recommended for the campaign.

Herczeg: I should like to present a very brief report of a spectroscopic investigation of $\beta$ Lyr which is in progress at Hamburg Observatory. This is not part of the 1971 international campaign. The spectra were taken in 1969 and 1972 with the $1 \mathrm{~m}$ telescope (blue region, dispersion $12 \AA \mathrm{mm}^{-1}$ at $\mathrm{H} \gamma$ ). Our intention was to measure some emission features ( $\mathrm{H} \delta, \mathrm{H} \gamma, \mathrm{He} \mathrm{I}$ 24472), their shape and, if possible, intensity in absolute units. There is surprisingly little known about the long-term behaviour of the emission lines in the spectrum of $\beta$ Lyr and a remark of Otto Struve that they may show a slow secular decline seemed to be interesting enough to follow up. We hope that a comparison of our recent spectra with Miss Gill's earlier observations and with the evaluation by Svolopoulos of Hamburg spectra taken in 1962 can possibly shed some light 
on this question. The 1972 spectra and part of the 1969 spectra are not yet fully reduced, but a preliminary inspection did not reveal a substantial secular change of the lines, although there may be a small decline.

Batten: I'd like to say that these results that have just been presented are the first from the international campaign that we organized last year, and there are several things of interest about them. I'd like to draw attention in particular to the very much shallower eclipse in the $\mathrm{H} \alpha$ emission lines found by Cester, and also to the fact that the asymmetry of the light curve is in opposite senses for the $\mathrm{H} \alpha$ emission line and the helium emission line at 26678 . It appears that the $\mathrm{H} \alpha$ emission was unusually strong last year during the time of the campaign. This was not our deliberate choice: it was a bonus! We seem to have organized the campaign just when the star was behaving in some unusual way. There is some evidence that this summer the $\mathrm{H} \alpha$ emission is weaker, and I think that when we get all the results from this campaign together, we may find that we have got something very interesting indeed.

Fracastoro: Does $\beta$ Lyr at any time behave in the usual way?

Herczeg: I saw Professor Hack less than two weeks ago, and she showed me her spectrograms and discussed $\beta$ Lyr with me. She mentioned that she had seen no clear indication of those peaks on the emission lines which are sometimes reported as showing velocity shifts opposite in phase from those of the absorption lines. The amplitude of the emission-line velocities is supposed to be about half that of the absorption-line velocities and this would indicate that the mass ratio of the B-type star to the secondary component is about one-half. How sure can we be that these peaks are real and indicate this motion? Perhaps it would be interesting to look at some of the early spectrograms obtained by Belopolsky and Campbell. I don't doubt that the secondary component is indeed the more massive since we have a good indication of the distance and absolute brightness and, consequently, of the size of the B8 star; combining this with the photometrically determined value of $R_{1} / a$ and the observed value of $a \sin i$, we obtain a mass ratio below unity.

Batten: A few years ago, Dr. Sahade and I studied $\mathrm{H} \alpha$ plates that have been obtained at Victoria from about 1966 onward. We couldn't construct a reasonable line profile, except on the assumption that there were two components to the emission a broad one, and a sharp one which we tentatively identified with emission from around the secondary star. Perhaps Dr. Sahade will say something more.

Sahade: I had not planned to talk during the sessions, but to listen and to think of what I will say on Tuesday, but I feel I should say something about this. Belopolsky and Curtiss measured the emission line at $\mathrm{H} \beta$, and apparently they found that this emission shifted in anti-phase with the stellar absorption lines. Examination of blueregion plates obtained at Mount Wilson in 1955 indicated the presence of an 'emission peak' at $\mathrm{H8}$, which also appears to shift in anti-phase with respect to the stellar absorption lines. More recent work on red-region plates by Batten and myself, however, showed that the emission profiles are badly affected by superposed absorption from the stellar and shell lines. The whole emission feature at $\mathrm{H} \alpha$, for instance, can be described, as Dr. Batten has just mentioned, by the superposition of 
two profiles. The broader one appears to change very little, or not at all; the narrower and stronger one appears to change greatly. The so-called 'emission peak' has no physical meaning - and I am to be blamed for having introduced such a description it results from blending of the narrower emission with absorption features.

O. C. Wilson: We have time for one more topic. Dr. Hutchings, would you like to present some comments on emission lines?

Hutchings: Hot gas streams and envelopes exist in several binary systems and give rise to strong Balmer emission lines. Profiles from such phenomena are essentially determined by the geometry and mass-motions of the streams and envelopes and in systems where these are eclipsed, we can derive information leading to an empirical picture of what the set-up looks like. From there on we can perhaps explain in more detail the theoretical implications.

The example I have in mind is the series of profiles of $\mathrm{H} \alpha$ in the spectrum of VV Cep, through eclipse, which Dr. Wright and I worked on recently. We assumed as a first approximation (which has subsequently been strengthened) an undisturbed out-ofeclipse emission profile. As the emission-line region is eclipsed, we observed the velocity distortion effect, well known in absorption lines in Algol systems, and other effects, as follows. The emission region is a disk or shell surrounding the hot star, in which rotational velocity is a function of radial distance. Thus, as the outermost, slowly rotating regions are initially eclipsed, we observe a dimunition in the innermost part of the emission profile. Towards central eclipse, the rapidly rotating (line-wing) section of the region became occulted. This sort of information gives us a good picture of the gross physical parameters describing the disk, and possibly the stream in systems of this sort. As another example I can quote the system AR Pav which Dr. Thackeray described briefly yesterday. Here we were able to derive similar information, unfortunately less detailed because of the necessarily low dispersion of the spectrographic material. Other systems of this type might well be observed in the same way. (The further example of $\beta$ Lyr comes to mind, although here we are probably hampered by many other horrendous complications.)

Cowley: I would like to mention two stars of the VV Cep type which particularly need photometry. They are AZ Cas and HR 2902 (= Boss 1985=KQ Pup). Both stars show absorption lines from an early B-type star in the ultra-violet, so that it should be possible to derive the mass ratio of both systems spectroscopically. AZ Cas has the shortest period (9.73) of any of the systems like VV Cep (which contain an $\mathrm{M}$ supergiant primary, a B star, and an extended shell which emits forbidden emission lines). There is almost no photometric information on its eclipses. The next one will occur sometime in 1975. HR 2902 has a very long period (277 days) but recent changes in the ultraviolet spectrum suggest a deep atmospheric eclipse is underway. If this is not a grazing eclipse, one might expect the possible photometric minimum within the next year. Because of the excessively long period it would be unfortunate not to observe the system now.

Hall: Why do they have variable-star names? Are they variable outside eclipses? Cowley: Yes. 
Andersen: Would uvby photometry be useful?

Cowley: Sure.

$O$. C. Wilson: I think we should call the meeting to a close.

\section{References}

Conti, P.: 1972, Astrophys. J. Letters 174, L79

Conti, P. and Alschuler, W. R.: 1971, Astrophys. J. 170, 325.

Fernie, J. D., Hube, J. O., and Schmidt, J. L.: 1968, Comm. 27 IAU Inf. Bull. Var. Stars, No. 263. Hack, M.: 1957, Publ. Astron. Soc. Pacific 69, 389.

Iben, I.: 1965, Astrophys. J. 141, 993.

Kuiper, G. P., Struve, O., and Strömgren, B.: 1937, Astrophys. J. 86, 570.

Larson, R. B.: 1969, Monthly Notices Roy. Astron. Soc. 145, 271.

Menzel, D. H.: 1939, Pop. Astron. 47, 6.

Sahade, J.: 1964, Trans. IAU XIIB, 494.

Sanford, R. F.: 1951, Astrophys. J. 113, 299.

Struve, O. and Pillans, H.: 1957, Publ. Astron. Soc. Pacific 69, 169.

Walborn, N. R.: 1971, Astrophys. J. Suppl. Ser. 23, 257.

Worrall, G. and Wilson, A. M.: 1972, Nature 236, 15.

Wright, K. O.: 1970, in A. Beer (ed.), Vistas in Astronomy 12, Pergamon Press, Oxford, p. 147.

Wright, K. O. and Kushwaha, R. S.: 1957, Publ. Astron. Soc. Pacific 69, 402. 